\title{
Class III malocclusion: an argument for early orthodontic treatment Eric Moe ${ }^{1}$
}

${ }^{1}$ University of Pittsburgh, School of Dental Medicine

\section{Abstract}

Class III malocclusion is a complex multifactorial condition with many genetic and environmental influences. Most often the condition is inherited in a Mendelian autosomal dominant pattern. Early referral and treatment can lead to better outcomes in orthodontic therapy. The subject presented for an early orthodontic referral at age 5.5 and showed signs of future Class III malocclusion. A rapid maxillary expander was given as interventional treatment for one year. At age 11.5 the subject was treated with braces for 2 years. The outcome was a normal Class I occlusion. The subject's mother had Class III malocclusion but was not evaluated early and was only able to establish an edge-toedge Class III malocclusion as the best treatment outcome without orthognathic surgery. The subject's grandmother was also Class III, establishing an autosomal dominance pattern of inheritance in the family. This case demonstrates the importance of the general dentist educating families about malocclusion and making orthodontic referrals as early as possible so the best treatment outcomes can be reached through orthodontic therapy.
Citation: Moe, E. (2017) Class III malocclusion: an argument for early orthodontic treatment. Dentistry 3000. 1:a001

doi:10.5195/d3000.2017.77

Received: May 22, 2017

Accepted: May 26, 2017

Published: June 19, 2017

Copyright: (02017 Moe, E. This is an open access article licensed under a Creative Commons Attribution Work 4.0 United States License.

Email: edm27@pitt.edu

\section{Introduction}

Malocclusion is the wideranging diagnosis given when the dentition from the two arches occlude, or come together, abnormally. The effects of malocclusion can include decreased masticatory function, compromised esthetics, and heightened risk of dental trauma [1]. Depending on the severity of the malocclusion, the overall effect is that quality of life can be compromised [1]. One of the most common classification systems of malocclusion is Angle's classification which designates malocclusion into three categories: Class I, II, and III. This case report focuses on Class III malocclusion, which is defined as the mesiobuccal cusp of the maxillary first permanent molar occluding distally to the buccal groove of the mandibular first molar, instead of aligning with it. Class III malocclu- sion can result in anterior crossbite and negative overjet or an edge-to-edge incisal relationship. It is estimated that up to $4 \%$ of European-Americans and $23 \%$ of Eastern Asians are affected by Class III malocclusion [2]. The condition can be diagnosed from a young age and it often becomes more evident with age and is one of the main reasons for orthodontic treatment or orthognathic surgery [2]. A better understanding of the genetic and environmental etiology of this condition could lead to earlier and more effective treatment options for those affected.

\section{Class III malocclusion is a} diverse and complex phenotype that can have several etiologies. The Class III phenotype is often described as mandibular prognathism, maxillary retrognathism, or a combination of both. One study analyzing lateral cephalometric variables further described the Class III phenotype into five clinically meaningful phenotypes [3]: combination of mild maxillary retrognathism and mandibular prognathism with a flat mandibular plane, combination of mild maxillary retrognathism and mandibular prognathism with a normal mandibular plane, large mandible expressed vertically, severe mandibular prognathism, and severe maxillary retrognathism [3]. The ability to classify the phenotypes associated with Class III malocclusion is a good step towards discovering the genetic etiology of each phenotype [3]. With the complexity of development and the numerous ways Class III malocclusion can occur, it is reasonable that Class III malocclusion is considered a multifactorial condition with both genetic and environmental influences. (c) BY ULLS D-Sorfe
New articles in this journal are licensed under a Creative Commons Attribution 4.0 United States License.

This journal is published by the University Library System, University of Pittsburgh as part of its D-Scribe Digital Publishing Program and is cosponored by the University of Pittsburgh Press. 


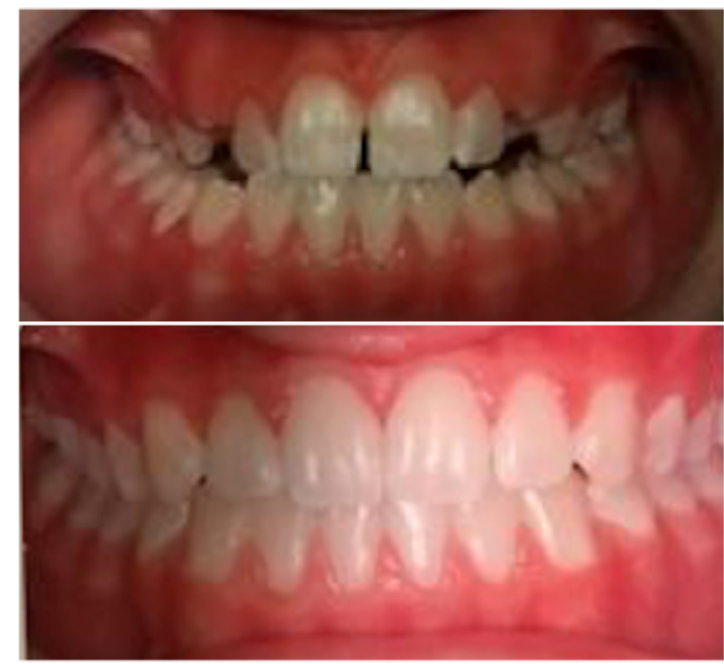

Figure 1. Subject before and after fixed orthodontic treatment at 11.5 and 13.5 years of age.

The etiology of Class III malocclusion is multifactorial and involves different genes and environmental factors. Different chromosomal locations and genes have been associated with Class III malocclusion in different ethnic populations [2]. Family studies also support a polygenic multifactorial inheritance pattern, as $13 \%$ of siblings of subjects with Class III malocclusion also exhibited Class III malocclusion [4]. Other studies analyzing twins have found that the rate of mandibular prognathism in monozygotic twins is six times higher than dizygotic twins, also suggesting polygenic inheritance and etiology [4]. However, pedigree analysis of Class III malocclusion in families has followed an autosomal dominant Mendelian pattern of inheritance with incomplete penetrance in a majority of analyses [2]. The conclusion of many of these studies is that Class III malocclusion within families is explained by some dominant gene being inherited in an autosomal dominant pattern, while likely be- ing modified by other genes and environmental influences [2].

Unfortu-

nately, while Class III malocclusion can often be described as a Mendelian autosomal dominant inherited condition, studies have already located many genes and chromosomal regions that are associated with this condition, showing that its genetic etiology is still very complex and not easily defined to one or two genes and not every case analyzed is easily generalized to all people of all ethnicities [2]. For example, analyses of Korean and Japanese families showed mandibular prognathism is associated with chromosome regions 1p36, 6q25, and 19q13.2 [5]. Colombian families have shown Class III malocclusion is associated with regions 1p22.1, $3 q 26.2,11 q 22,12 q 13.13$, and $12 q 23$ [6]. Analyses of Han Chinese people showed regions 14q24.3-q31.2 influence mandible growth [7]. An Estonian family was described as having the mutation p.Ser182Phe in DUSP6 leading to Class III malocclusion [2]. All of these cases show how Class III occlusion has a complex genetic etiology that is multifactorial and variable. Furthermore, there are environmental factors that may contribute to the development of Class III malocclusion. The mandibular bone is developed from condylar cartilage, and mechanical loading may influence the response of the condylar cartilage, leading to growth of the mandible through various growth factors or signaling molecules. These factors include Indian Hedgehog homolog, parathyroid-hormone like hormone, insulin-like growth factor-1, and vascular endothelial growth factor. It is possible that genes coding for these factors may express the factors at levels that have interactions with certain environmental forces at play that combine to predispose the development of a Class III phenotype [4].

In summary, there is much more that needs to be learned about the etiology of Class III malocclusion, but current knowledge shows there are many genes and environmental factors that can lead to the condition. Interestingly, while it has such a complex multifactorial genetic and environmental etiology, it more often than not follows an autosomal dominant Mendelian inheritance with incomplete penetrance that is likely influenced by other genes and environmental factors.

Because of the complex and poorly-understood etiology of Class III malocclusion, treatment plans focus on fixing symptoms instead of preventing etiology [1]. The two main concepts to consider for a treatment plan are its timing and type of appliance [8]. Often, early treatment can help prevent the need for future orthognathic surgery, so a fixed orthodontic treatment, like braces, is 


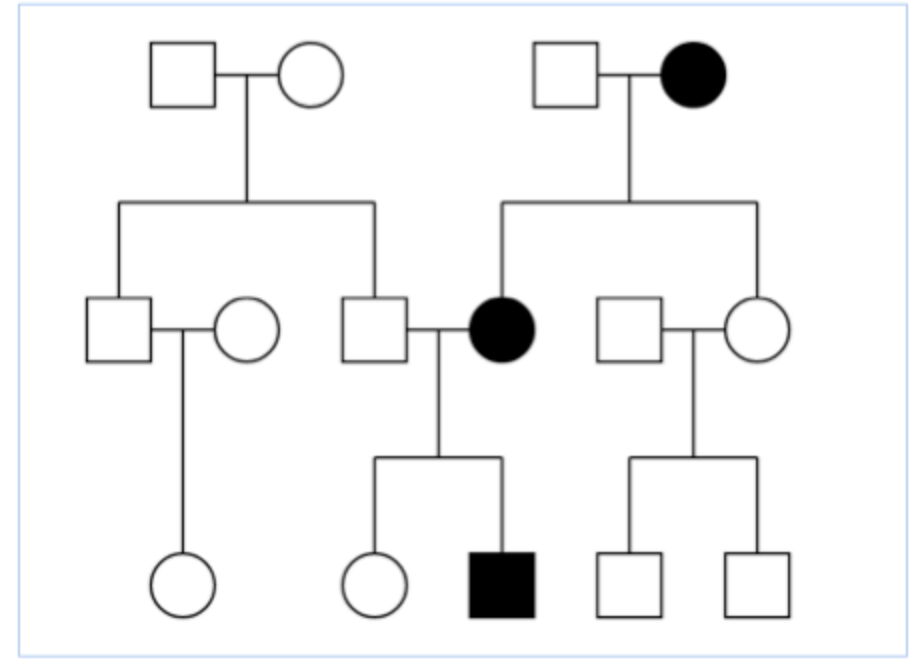

Figure 2. Pedigree for Class III malocclusion in the subject's family. The patient, his mother, and his grandmother had the condition.

sufficient instead. Currently, there is lacking evidence to support the long term benefits of early treatment, but short term evidence shows both skeletal and dental beneficial effects of early treatment in Class III malocclusion [8].

\section{Case Presentation}

The Angle's Class III malocclusion subject is a Caucasian male who first presented for orthodontic evaluation at 5.5 years of age. The subject presented with a probable Class III growth pattern that may become more obvious as growth continued. He had a complete anterior crossbite and normal amount of anterior overbite. He had moderate maxillary crowding and adequate mandibular arch length. The maxillary arch was constricted. The profile of the subject was prognathic. The subject received early interventional orthodontic treatment with a bonded rapid maxillary expander and reverse pull headgear at night to protract the maxilla for one year.
The subject was reevaluated after interventional treatment. He still showed a possible Class III growth pattern that may become more obvious as growth continues. However, the interventional treatment was successful because the subject had normal overjet, indicating the complete anterior crossbite was eliminated. The subject had adequate maxillary and mandibular arch length, indicating the moderate maxillary crowding was relieved. The evaluation indicated that comprehensive orthodontic treatment would be needed in the future and that the subject should be periodically reevaluated.

At 11.5 years of age the subject was evaluated to have an edge-to-edge incisal relationship. His mandibular midline was also shifted $1-2 \mathrm{~mm}$ to the right. The maxillary arch was constricted again. At this time, the subject received full fixed braces treatment to correct the edge-to-edge incisal relationship and other findings. The expected treatment duration was 24-30 months and the expected outcome was a Class I normal occlusion. After 24 months of treatment, the subject finished the course of treatment with a Class I normal occlusion at $\mathbf{1 3 . 5}$ years of age. Figure 1 shows before (age 11.5) and after (age 13.5) pictures of the subject's dentition.

Family history was gathered about the subject in terms of Class III malocclusion. The pedigree for all known relatives' occlusion before any orthodontic treatments is shown in Figure 2. The subject's mother and his grandmother on his mother's side also had Class III malocclusion. No one else in the family, including his sister, father, and all other relatives had Class III malocclusion.

\section{A limited dental history} was obtained from the subject's mother in regards to her Class III malocclusion. The mother had a similar Class III malocclusion to the subject, but she did not receive any orthodontic evaluation or interventional orthodontic treatment until she was 13 years of age. Her outlook at that time was either orthognathic surgery or a probable best case scenario of an edge-to-edge incisal relationship after braces treatment. She opted for braces and the result was the predicted edge-to-edge incisal relationship, which was better than the more severe Class III malocclusion at the beginning of treatment. No detailed history about the grandmother's Class III malocclusion or treatment could be obtained. 


\section{Discussion}

This case presents a pedigree with a Mendelian inheritance pattern of autosomal dominance as the subject, his mother, and his grandmother all have Class III malocclusion. While the pedigree available is a small sample size, in each case of inheritance, $50 \%$ of the children received the Class III malocclusion phenotype, which is to be expected with an autosomal dominant inheritance pattern. This type of inheritance pattern matches what the literature reports for Class III malocclusion being multifactorial in nature, but often inherited in an autosomal dominant manner. It is likely that if the subject has offspring, they will each have a $50 \%$ chance of developing a Class III malocclusion.

This case also demonstrates the importance of general dentists educating families with young children about malocclusion early on and making early referrals to orthodontists for evaluation so early interventional treatment can be provided, if needed. The subject of this case received an early referral at the age of 5.5 and received a rapid maxillary expander for one year before fixed appliance braces at 11.5 years of age for 2 years of treatment. It is likely that a normal occlusion was possible due to the interventional treatment received at a young age. This course of treatment can be compared to the subject's mother who did not receive any interventional treatment or evaluation at an early age. Her best non-surgical treatment outcome was a Class III edge-to-edge incisal relationship. This shows that dentists have the ethical implication to be knowledgeable and thorough in recognizing early malocclusion and making referrals. This includes the ethical necessity of educating the family about why following up with the orthodontic referral is important. The dentist is also in an advantageous position to start educating the family when the children are born about the possibilities of their child requiring orthodontic treatment in the future so they can begin to plan for this treatment as early as possible. The knowledgeable dentist will be able to educate families with parents that have Class III malocclusion that it is likely that their children may also have Class III malocclusion that requires treatment. It is also thorough to inform parents that had normal occlusion that malocclusion, and specifically Class III malocclusion, is complex and can be sporadic as well, so the parents know it is possible that orthodontic therapy may be required for their children even if they did not require it themselves. Overall, this case shows that early patient education and early orthodontic referral and treatment can help the patient achieve the best possible outcome, in this case a normal occlusion, when presenting with Class III malocclusion.

\section{Conclusion}

Class III malocclusion has several phenotype subgroups that result from a complex multifactorial etiology with many genes and environmental factors influencing its development. In many cases, Class III malocclusion is inherited in a Mendelian autosomal dominant pattern. Early treatment of Class III malocclusion can aid in reaching a normal occlusion, and dentists can utilize early patient education and orthodontic referrals to facilitate this early treatment. Special emphasis should be given to educate patients with parents who have or had Class III malocclusion, as they are most susceptible to the condition.

\section{References}

1. Moreno Uribe LM, Miller SF. Genetics of the dentofacial variation in human malocclusion. Orthod Craniofac Res. 2015 Apr;18 Suppl 1:91-9. doi: 10.1111/ocr.12083. PubMed PMID: 25865537; PubMed Central PMCID: PMC4418210.

\section{Nikopensius $T$, Saag $M$,} Jagomägi $T$, Annilo T, Kals M, Kivistik PA, Milani L, Metspalu A. A missense mutation in DUSP6 is associated with Class III malocclusion. J Dent Res. 2013

Oct;92(10):893-8. doi: 10.1177/0022034513502790. Epub 2013 Aug 21. Erratum in: J Dent Res. 2016 Jul;95(8):955. PubMed PMID: 23965468.

\section{Moreno Uribe LM, Vela KC,} Kummet C, Dawson DV, Southard TE. Phenotypic diversity in white adults with moderate to severe Class III malocclusion. Am J Orthod Dentofacial Orthop. 2013 Jul;144(1):32-42. doi: 10.1016/j.ajodo.2013.02.019. PubMed PMID: 23810043; Pub- 
Med Central PMCID:

PMC3964133.

4. Xue F, Wong RW, Rabie AB.

Genes, genetics, and Class III malocclusion. Orthod Craniofac Res. 2010 May;13(2):69-74. doi:

10.1111/j.16016343.2010.01485.x . Review. PubMed PMID:

20477965.

5. Yamaguchi T, Park SB, Narita A, Maki K, Inoue I. Genome-wide linkage analysis of mandibular prognathism in Korean and Japanese patients. J Dent Res. 2005 Mar;84(3):255-9. PubMed PMID: 15723866.

6. Frazier-Bowers S, RinconRodriguez R, Zhou J, Alexander K, Lange $E$. Evidence of linkage in a Hispanic cohort with a Class III dentofacial phenotype. J Dent Res. 2009 Jan;88(1):56-60. doi:

10.1177/0022034508327817. PubMed PMID: 19131318; PubMed Central PMCID:

PMC2777651.

7. Li Q, Li X, Zhang F, Chen F. The identification of a novel locus for mandibular prognathism in the Han Chinese population. J Dent Res. 2011 Jan;90(1):53-7. doi: 10.1177/0022034510382546. Epub 2010 Nov 1. PubMed PMID: 21041550.

8. Woon SC, Thiruvenkatachari B. Early orthodontic treatment for Class III malocclusion: A systematic review and meta-analysis. Am J Orthod Dentofacial Orthop. 2017 Jan;151(1):28-52. doi: 10.1016/j.ajodo.2016.07.017. Review. PubMed PMID: 28024779. 